\title{
The neglected gender - males in bees
}

On a first glance at a nest of social bees females are obviously the dominant sex, while males are mostly present only during a short season and do not to seem to participate in any of the colony tasks. This failure to catch attention is reflected in a conspicuous neglect of the male gender in scientific studies. However, males contribute to the fitness of their offspring as much as females do. Further their morphology and behavior are as important features for taxonomy and evolutionary theories as any other males in the animal kingdom. Thus the blind spot for the male sex misses an important component for the understanding of the social cycle of individual and colonial life.

This special issue wants to initiate more research in this interesting but badly neglected field. It contains original articles with new data as well reviews which arrange known facts and features into an evolutionary framework. It also includes solitary bees of the tribe Apoidea to contribute to the understanding of the relation between both sexes in a non-social status from which eusociality is thought to have evolved.

"The literary caricature of the idling drone is probably far from the truth. A more appropriate view is that of the frenetic male in desperate search of an elusive, receptive female (Paxton 2005, page 145)."

Gudrun Koeniger

Oberursel, January 2005

\section{Éditorial}

\section{Le sexe délaissé - les mâles d'abeilles}

Au premier coup d'œil, les femelles sont apparemment le sexe dominant chez les insectes sociaux, tandis que les mâles ne sont présents que pendant une courte saison et ne semblent pas prendre part aux diverses tâches de la colonie. Le peu d'attention que les mâles suscitent chez l'observateur se reflète dans le manque d'intérêt flagrant dans les recherches scientifiques. Pourtant les mâles contribuent autant que les femelles à la fitness de leur descendance. Par ailleurs, comme pour tous les autres mâles du règne animal, leur morphologie et leur comportement sont importants pour la taxonomie et les théories évolutionnistes. La tache aveugle pour le sexe masculin ignore donc une composante importante de la compréhension du cycle de développement de la vie individuelle et sociale.

Ce numéro spécial vise à stimuler plus de recherches dans ce domaine intéressant mais largement délaissé. Il comporte des articles originaux fournissant de nouvelles données, ainsi que des articles de synthèse qui présentent les faits connus d'un point de vue évolutionniste. Il inclut aussi les abeilles solitaires de la tribu des Apoidea pour contribuer à la compréhension de la relation entre les deux sexes dans un statut non social, à partir duquel l'eusocialité est sensée avoir évoluer.

Gudrun Koeniger

Oberursel, janvier 2005 


\section{Editorial}

\section{Das vernachlässigte Geschlecht - die Männchen der Bienen}

Auf den ersten Blick scheinen bei den sozialen Insekten die Weibchen das ganz offensichtlich dominierende Geschlecht zu sein - die Männchen sind meist nur über kurze Zeit während der Saison zu finden und beteiligen sich offenbar nicht an irgendwelchen Arbeiten innerhalb der Nester. Die geringe Aufmerksamkeit, die das männliche Geschlecht bei dem Betrachter erregt, geht mit einer augenfälligen Vernachlässigung in wissenschaftlichen Untersuchungen einher. Die Männchen tragen allerdings ebenso viel zur Fitness ihrer Nachkommen bei wie die Weibchen. Ihre Morphologie und ihr Verhalten sind von gleich großer Wichtigkeit für die Taxonomie und evolutionäre Theorien wie bei den anderen Männchen des Tierreiches. Der blinde Fleck für das männliche Geschlecht blendet daher einen für das Verständnis des sozialen und individuellen Lebenszyklus wichtigen Teilbereich aus.

Dieses Sonderheft zielt darauf ab, verstärkte Untersuchungen in diesem interessanten, aber stark vernachlässigten Bereich anzuregen. Es enthält eine Reihe von Originalbeiträgen mit neuen Untersuchungen sowie von Übersichtsartikeln, die bekannte Tatsachen in eine evolutionäre Sicht einordnen. Es schließt weiterhin auch einzellebende Bienen des Tribus Apoidea ein, um aus dem Blickwinkel eines mutmaßlich der Euozialität vorangehenden nichtsozialen Status zum Verständnis des Verhältnisses zwischen beiden Geschlechtern beizutragen.

Gudrun Koeniger

Oberursel, Januar 2005 